DOI: $10.21105 /$ joss.01296

\section{Software \\ - Review ¿ \\ - Repository u \\ - Archive [}

Submitted: 23 February 2019 Published: 01 May 2019

\section{License}

Authors of papers retain copyright and release the work under a Creative Commons Attribution 4.0 International License (CC-BY).

\title{
PoreSpy: A Python Toolkit for Quantitative Analysis of Porous Media Images
}

\section{Jeff T. Gostick ${ }^{1}$, Zohaib A. Khan ${ }^{1}$, Thomas G. Tranter ${ }^{1,2}$, Matthew D.R. Kok ${ }^{2,3}$, Mehrez Agnaou ${ }^{1}$, Mohammadamin Sadeghi ${ }^{3}$, and Rhodri Jervis $^{2}$}

1 Department of Chemical Engineering, University of Waterloo, Waterloo, ON, Canada 2 Department of Chemical Engineering, University College London, London, United Kingdom 3 Department of Chemical Engineering, McGill University, Montreal, QC, Canada

\section{Summary}

Porous materials play a central role in many technologies, from micron-thick electrodes used in batteries and fuel-cells (Karan, 2017) to kilometer-long geologic formations of interest in oil recovery, contaminant transport and CO2 sequestration (Kumar et al., 2005). These applications share a common interest in analyzing the transport processes occurring at the pore-scale, since these ultimately control the observable macroscopic behavior (Anovitz \& Cole, 2015). Images of porous materials are a valuable source of information, since performance and structure are intimately linked through the topology and geometry of the media. A variety of techniques are available for imaging a material's internal pore structure with sub-micron resolution, including X-ray tomography (Coles et al., 1998), ptychography (Dierolf et al., 2010), and FIB-SEM sectioning (Heaney, Vicenzi, Giannuzzi, \& Livi, 2001). Each of these tools can provide exquisitely detailed images, and retrieving quantitative information from these images has become a vital tool in all areas of porous media research.

The analysis of images, both 2D sections and 3D volumetric data (Taiwo et al., 2016), is an established field, dating to the advent of computers with sufficient memory to store and manipulate the rather large file sizes. Pioneering work by Torquato, summarized in his textbook (Torquato, 2005), laid a solid foundation by defining many metrics still widely used, including chord-length distribution (Jervis et al., 2018) and radial-density function (Kok, Jervis, Brett, Shearing, \& Gostick, 2018). The field is far from dormant, however, as increasingly powerful computers allow application of concomitantly advanced algorithms, such as watershed partitioning to create networks (Khan, Tranter, Agnaou, Elkamel, \& Gostick, 2019, J Gostick (2017)) for pore-network modeling (J. Gostick et al., 2016), and direct numerical simulations (García-Salaberri et al., 2019) which can often supplant experiments (García-Salaberri et al., 2018) or even conduct experiments that are not physically possible (García-Salaberri, Hwang, Vera, Weber, \& Gostick, 2015, M. D. R. Kok et al. (2019)). The aim of PoreSpy is to collect the various tools and functions needed by researchers to perform quantitative analysis of volumetric images of porous materials into a single, easy to use, open-source package. PoreSpy is written in the popular Python language which contains several image processing libraries offering the full suite of necessary tools (Gouillart, Nunez-Iglesias, \& Walt, 2016, Walt et al. (2014)). PoreSpy contains a large, though not exhaustive, set of the most commonly used tools for porous media analysis, which typically work by applying a series of the basic image analysis functions with appropriate arguments and adjustments. The functions in PoreSpy are organized into the following categories: 
- generators: create artificial images

- filters: highlight features of interest

- metrics: extract quantitative information

- networks: pore-network representations

- tools: helper functions

- io: convert between formats

- visualization: create basic views

The scope of PoreSpy is constrained to tools for the analysis and manipulation of images. As such it does not attempt to offer 3D visualization which is already available in opensource software such as Paraview (Ayachit, 2015) and numerous sophisticated commercial packages (e.g. DragonFly, Avizo). PoreSpy also stops short, at least for now, of performing direct numerical simulations on images, despite this being a common and powerful use of volumetric images, since such algorithms are extremely complex and best handled by dedicated packages. Instead, PoreSpy offers tools for converting images into file formats suitable for these other packages.

Being implemented in Python means it can be installed with a single command (pip in stall porespy) without any compilation, and is accessible to researchers with any level of programming experience via Jupyter notebooks. The design of PoreSpy is modelled after other related packages in the Scipy stack, as a collection of simple functions with well-defined and clearly documented inputs and outputs. It is open-source so that other researchers can confidently use each function knowing how it works, but also so that other researchers can contribute new algorithms or Python-implementations of seminal tools.

\section{Acknowledgements}

MDRK acknowledges support from EPSRC under grant EP/R020973/1. MDRK and RJ acknowledge the support of the Faraday Institution Degradation and Multi scale Modelling projects. ZA express his appreciation to the University of Engineering and Technology Lahore, Pakistan for their funding and support.

\section{References}

Anovitz, L. M., \& Cole, D. R. (2015). Characterization and Analysis of Porosity and Pore Structures. Reviews in Mineralogy and Geochemistry, 80(1), 61-164. doi:10.2138/ rmg.2015.80.04

Ayachit, U. (2015). The ParaView Guide: A Parallel Visualization Application. Kitware, Inc. Retrieved from http://dl.acm.org/citation.cfm?id=2789330

Coles, M. E., Hazlett, R. D., Spanne, P., Soll, W. E., Muegge, E. L., \& Jones, K. W. (1998). Pore level imaging of fluid transport using synchrotron X-ray microtomography. Journal of Petroleum Science and Engineering, 19(1), 55-63. doi:10.1016/S0920-4105(97) 00035- 1

Dierolf, M., Menzel, A., Thibault, P., Schneider, P., Kewish, C. M., Wepf, R., Bunk, O., et al. (2010). Ptychographic X-ray computed tomography at the nanoscale. Nature, 467(7314), 436-439. doi:10.1038/nature09419

García-Salaberri, P. A., Hwang, G., Vera, M., Weber, A. Z., \& Gostick, J. T. (2015). Effective diffusivity in partially-saturated carbon-fiber gas diffusion layers: Effect of through-plane saturation distribution. International Journal of Heat and Mass Transfer, 86, 319-333. doi:10.1016/j.ijheatmasstransfer.2015.02.073 
García-Salaberri, P. A., Zenyuk, I. V., Hwang, G., Vera, M., Weber, A. Z., \& Gostick, J. T. (2019). Implications of inherent inhomogeneities in thin carbon fiber-based gas diffusion layers: A comparative modeling study. Electrochimica Acta, 295, 861-874. doi:10.1016/ j.electacta.2018.09.089

García-Salaberri, P. A., Zenyuk, I. V., Shum, A. D., Hwang, G., Vera, M., Weber, A. Z., \& Gostick, J. T. (2018). Analysis of representative elementary volume and throughplane regional characteristics of carbon-fiber papers: Diffusivity, permeability and electrical/thermal conductivity. International Journal of Heat and Mass Transfer, 127, 687-703. doi:10.1016/j.ijheatmasstransfer.2018.07.030

Gostick, J. (2017). Versatile and efficient pore network extraction method using marker-based watershed segmentation. Physical Review E, 96(2), 023307. doi:10.1103/PhysRevE.96.023307

Gostick, J., Aghighi, M., Hinebaugh, J., Tranter, T., Hoeh, M. A., Day, H., Spellacy, B., et al. (2016). OpenPNM: A Pore Network Modeling Package. Computing in Science Engineering, 18(4), 60-74. doi:10.1109/MCSE.2016.49

Gouillart, E., Nunez-Iglesias, J., \& Walt, S. van der. (2016). Analyzing microtomography data with Python and the scikit-image library. Advanced Structural and Chemical Imaging, 2(1), 18. doi:10.1186/s40679-016-0031-0

Heaney, P., Vicenzi, E., Giannuzzi, L., \& Livi, K. (2001). Focused ion beam milling: A method of site-specific sample extraction for microanalysis of Earth and planetary materials. American Mineralogist, 86(9), 1094-1099. doi:10.2138/am-2001-8-917

Jervis, R., Kok, M. D. R., Montagut, J., Gostick, J. T., Brett, D. J. L., \& Shearing, P. R. (2018). X-ray Nano Computed Tomography of Electrospun Fibrous Mats as Flow Battery Electrodes. Energy Technology, 6(12), 2488-2500. doi:10.1002/ente.201800338

Karan, K. (2017). PEFC catalyst layer: Recent advances in materials, microstructural characterization, and modeling. Current Opinion in Electrochemistry, 5(1), 27-35. doi:10. 1016/j.coelec.2017.08.018

Khan, Z. A., Tranter, T., Agnaou, M., Elkamel, A., \& Gostick, J. (2019). Dual network extraction algorithm to investigate multiple transport processes in porous materials: Image-based modeling of pore and grain scale processes. Computers \& Chemical Engineering, 123, 64-77. doi:10.1016/j.compchemeng.2018.12.025

Kok, M. D. R., Jervis, R., Brett, D., Shearing, P. R., \& Gostick, J. T. (2018). Insights into the Effect of Structural Heterogeneity in Carbonized Electrospun Fibrous Mats for Flow Battery Electrodes by X-Ray Tomography. Small, 14(9), 1703616. doi:10.1002/ smll.201703616

Kok, M. D. R., Jervis, R., Tranter, T. G., Sadeghi, M. A., Brett, D. J. L., Shearing, P. R., \& Gostick, J. T. (2019). Mass transfer in fibrous media with varying anisotropy for flow battery electrodes: Direct numerical simulations with 3D X-ray computed tomography. Chemical Engineering Science, 196, 104-115. doi:10.1016/j.ces.2018.10.049

Kumar, A., Noh, M. H., Ozah, R. C., Pope, G. A., Bryant, S. L., Sepehrnoori, K., \& Lake, L. W. (2005). Reservoir Simulation of $\mathrm{CO}_{2}$ Storage in Aquifers. SPE Journal, 10(03), 336-348. doi:10.2118/89343-PA

Taiwo, O., Finegan, D., Eastwood, D., Fife, J., Brown, L., Darr, J., Lee, P., et al. (2016). Comparison of three-dimensional analysis and stereological techniques for quantifying lithium-ion battery electrode microstructures. Journal of Microscopy, 263(3), 280-292. doi:10.1111/jmi.12389

Torquato, S. (2005). Random Heterogeneous Materials: Microstructure and Macroscopic Properties. Springer Science \& Business Media. 
Walt, S. van der, Schönberger, J. L., Nunez-Iglesias, J., Boulogne, F., Warner, J. D., Yager, N., Gouillart, E., et al. (2014). Scikit-image: Image processing in Python. PeerJ, 2, e453. doi:10.7717/peerj. 453 